\title{
Research of nitric acid solutions neutralization process with ammonia on the model of a periodic action apparatus
}

\author{
C Ilia Yu. Sakharov, ${ }^{1}$ Alexsey F. Makhotkin, ${ }^{2}$ Yury Yu. Sakharov, ${ }^{3 *}$ \\ Igor A. Makhotkin, ${ }^{4}$ Roman V. Durnik, ${ }^{5+}$ and Rauf F. Sabirov ${ }^{6}$ \\ ${ }^{1}$ Chemical Plant Equipment Department. Kazan National Research Technological University. \\ K. Marks St., 68. Kazan, 420015.Russia.E-mail:wisefellow@mail.ru; \\ 2ohz.kstu@rambler.ru; ${ }^{3} u$ sacharas@mail.ru; ${ }^{4}$ oxzkstu@kstu.ru; ${ }^{5}$ durnikroman1@gmail.co; \\ 6Sabirov.9090@mail.ru.Phone: ${ }^{3}+7$ (905)314-42-39; ${ }^{5}+7$ (960) 033-94-35.
}

\section{*Supervising author; ${ }^{+}$Corresponding author}

Keywords: nitric acid, ammonia, ammonium nitrate, parameters, process research, device, technology.

\begin{abstract}
Research of nitric acid aqueous solution neutralization process with ammonia gas were performed on model of a periodic action apparatus. This model is implemented on a fundamentally new laboratory unit for analyzing the process of absorption in a gas-liquid system and includes a measuring tank containing selected gas, which is installed vertically and a measuring container with selected liquid, which is connected to the measuring gas container from below. During the process, the numerical values of the piston position are fixed according to the measured time scale and the kinetic curves of the process are constructed, these curves are processed using known algorithms and a conclusion is made about the physicochemical (kinetic and diffusion) process parameters. Investigations have shown that the process of nitric acid aqueous solutions neutralization with ammonia practically does not depend on the concentration of nitric acid in the liquid phase and is directly proportional to the concentration of ammonia in the gas phase. Analysis of the kinetic curves, taking into account the fact that the chemical reaction between ammonia and nitric acid proceeds almost instantly, allows us to make an assumption about the mechanism of ammonia absorption by aqueous solutions of nitric acid - as a diffusion process, the limiting stage of which is the diffusion of ammonia in a thin film of gas at the phase contact surface. At the same time, growth of the phases contact surface area and the rate increase of surface renewal are the main tasks for intensifying the process. A method is proposed to intensify the process of ammonia absorption by nitric acid solutions based on using vortex apparatus with a high degree of gas turbulization and a rapid renewal of the phases contact surface.
\end{abstract}

\section{References}

[1] I.Yu. Sakharov [and etc.]. Regularities of the kinetics of absorption of ammonia by nitric acid in the conditions of production of ammonium nitrate. Bulletin of Kazan Technological University. 2013. Vol.16. No.14. P.74-76. (russian)

[2] Device for determining the volume of gas involved in the mass transfer process in the gas-liquid system: US Pat. 2659462 Ros. Federation: IPC G 01 N 7/04 / Sakharov Yu.N., Makhotkin A.F., Sakharov I.Yu., Makhotkin I.A.; applicant and patentee Federal State Budgetary Educational Institution of Higher Education "Kazan National Research Technological University". No. 2017122505, 06/26/2017; publ. 02.07.2018. 2p.: ill.

[3] K.F. Pavlov, P.G. Romankov, A.A. Noskov. Examples and tasks for the course of processes and devices of chemical technology. Leningrad: Chemistry. 1987. P.291. (russian) 